\title{
ПРАКТИКА ПРЕДИКТИВНОЙ ДИАГНОСТИКИ В ОЦЕНКЕ РИСКА РАЗВИТИЯ САХАРНОГО ДИАБЕТА 2 ТИПА
}

\author{
Заботин М.B.
}

ООО «Базис Геномик», Новосибирск

\begin{abstract}
ЦЕЛЬ: выявить статистическое различие в полиморфизмах между гаплогруппой R1b и большой статистической группой жителей России.
\end{abstract}

МАТЕРИАЛЫ И МЕТОДЫ: около 12000 записей исходной базы данных генетической лаборатории исследований, выполненных с 2016 по 2020 гг.

\section{Лабораторные технологии.}

Метод аллель-специфичной ПЦР в реальном времени с флуоресцентно меченными TaqMan-зондами на приборах DT-Prime (ДНК-Технология, Москва). Праймеры подобраны на основании данных публичных ресурсов Vector NTI v11.5 (Invitrogen). Синтез олигонуклеотидов выполнялся ООО «ДНК Синтез» (Москва).

Оценка результатов: сопоставление частот признаков в популяционных выборках (\%оо и коэффициенты различий). Непараметрический анализ $\left(\mathrm{X}^{2}\right)$. Используемое программное обеспечение MS Excel 365, SPSS 17,0 .

РЕЗУЛЬТАТЫ: был выявлен ряд статистически значимых различий рисков сахарного диабета 2 типа в сравнении с европейской гаплогруппой R1b.

Выводы: выявленные полиморфизмы риска развития сахарного диабета 2 типа позволяют построить персонифицированную программу превентивных мероприятий для конкретного пациента. Статистика выявленных полиморфизмов характеризует достаточно высокий риск развития сахарного диабета 2 типа и метаболического синдрома среди населения Российской Федерации.

Численность пациентов с диагнозом «сахарный диабет» выросла за 2019 г. среди взрослых - на 4,7\%, среди детей — на 5,3\%. На начало 2020 г. было зарегистрировано 5,1 млн заболеваний сахарным диабетом у взрослого населения в России и почти 50 тысяч - у детей. Негативные тренды наблюдаются и в общем увеличении количества и кумулятивном накоплении пациентов, о чем свидетельствует динамика размеров диспансерных групп.

Современные исследования генома человека и идентификация генов, полиморфизмы в которых предрасполагают к метаболическим изменениям, в сопоставлении с отработанными подходами клинической и лабораторной диагностики позволяют с высокой точностью выявлять группы населения с высоким риском развития мультифакториальных заболеваний. Предиктивная диагностика, учитывающая генетическую предрасположенность к развитию сахарного диабета 2 типа, для таких групп населения позволяет сформировать комплекс превентивных и лечебно-профилактических мероприятий, направленных на снижение степени риска под контролем врача.

Для оценки распространенности основных маркеров генетического риска развития сахарного диабета в Российской популяции был проведен анализ данных генетического тестирования и анкетных данных об индивидуальных особенностях людей, прошедших тестирование: пол, возрастная группа, особенности питания и физической нагрузки, наследственный анамнез, состояние здоровья и элементы образа жизни 11625 жителей РФ (на основании данных Регистра генетических исследований компании BGG 2016-2021 гг.) из разных регионов в сравнении с европейскими индикаторами. Был выявлен ряд статистически значимых различий по сравнению с европейской гаплогруппой R1b. Сочетание гомо- и гетерозиготных аллелей в генах FTO, SLC30A8, TCF7L2, KCNJ11, связанных с риском развития сахарного диабета 2 типа в европейской гаплогруппе R1b выше, чем в группе жителей России. Но даже в этом случае гомо- и гетерозиготные аллели в генах FTO, SLC30A8 и KCNJ11, связанные с высоким риском развития сахарного диабета, выявляются достаточно часто.

Знание о генетических рисках населения является важнейшим этапом в организации превентивной диагностики и построении комплексных оздоровительных и профилактических программ, помогающих снизить вероятность развития сахарного диабета и метаболического синдрома. Выполнение генетического тестирования позволяет на объективных основаниях построить персонифицированную программу превентивных мероприятий, направленных на сохранение здоровья и активное долголетие конкретного человека и групп населения.

КЛЮЧЕВЫЕ СЛОВА: сахарный диабет; полиморфизм; геном; генетика. 